\title{
Copper Loss and Torque Ripple Minimization in Switched Reluctance Motors Considering Nonlinear and Magnetic Saturation Effects
}

\author{
Milad Dowlatshahi ${ }^{\dagger}$, Sayed Morteza Saghaiannejad*, Jin-Woo Ahn ${ }^{* *}$, and Mehdi Moallem** \\ $\dot{\dagger}^{*}$ Dept. of Electrical and Computer Eng., Isfahan University of Technology, Isfahan, Iran \\ ** Dept. of Mechatronics Eng., Kyungsung University, Busan, Korea
}

\begin{abstract}
The discrete torque generation mechanism and inherently nonlinear magnetic characterization of switched reluctance motors lead to unacceptable torque ripples and limit the application of these motors. In this study, a phase current profiling technique and torque sharing function are proposed in consideration of magnetic saturation effects and by minimizing power loss in the commutation area between the adjacent phases. Constant torque trajectories are considered in incoming and outgoing phase current planes based on nonlinear $T$-i-theta curves obtained from experimental measurements. Optimum points on constant torque trajectories are selected by improving drive efficiency and minimizing copper loss in each rotor position. A novel analytic invertible function is introduced to express phase torque based on rotor position and its corresponding phase current. The optimization problem is solved by the proposed torque function, and optimum torque sharing functions are derived. A modification method is also introduced to enhance the torque ripple-free region based on simple logic rules. Compared with conventional torque sharing functions, the resultant reference current from the proposed method has less peak and effective values and exhibits lower copper loss. Experimental and simulation results from a four-phase $4 \mathrm{KW} 8 / 6 \mathrm{SRM}$ validate the effectiveness of the proposed method.
\end{abstract}

Key words: Copper loss, Magnetic saturation effects, Switched reluctance motors, Torque ripple-minimization

\section{INTRODUCTION}

Switched reluctance machines (SRMs) are constructed with a double salient motor and have no winding or permanent magnets in the rotor. They have a rugged and fault tolerant structure with the most robust and reliable construction [1]. Considering their simple structure, low manufacturing and repair costs, and ability to operate in a wide range of temperature and speed, SRMs have received much attention recently. Higher torque ripples compared with other AC-type machines is one of the most critical drawbacks of SR applications [2]. The torque pulsation mechanism and highly saturated nature of magnetic characterization lead to undesirable torque ripples, unfavorable acoustic noises, and deterioration in drive performance, especially in high-precision industrial applications. Many investigations have been

Manuscript received Aug. 31, 2013; accepted Dec. 19, 2013 Recommended for publication by Associate Editor Dong-Hee Lee. ${ }^{\dagger}$ Corresponding Author: dolatshahi@ec.iut.ac.ir

Tel: +98-311-3912450, Fax: +98-311-3912451, Isfahan Univ. of Tech.

*Dept. of Electrical and Com. Eng., Isfahan Univ. of Technology, Iran

** Dept. of Mechatronics Eng., Kyungsung Univ., Busan, Korea undertaken and many torque control techniques have been introduced recently to suppress torque ripples [3]-[9]. Although design approaches were considered in [3] and [4] to produce constant torque and torque ripples were minimized, control techniques continue to be investigated to maintain motor torque at a desired level in conventional existing SRMs. One of the most convenient approaches is to coordinate the incoming and outgoing phase torque so that the resultant torque remains constant during the commutation between adjacent phases. Torque sharing function (TSF) is the most well-known torque control method in which reference torques for individual phases are defined assuming that total torque is maintained at a constant level. Direct instantaneous torque control also involves a simple torque control concept [10]. However, the implementation of switching rules and advanced hysteresis functions is complicated. Some conventional TSFs, such as linear, cosine, cubic, and exponential types, have been reported in literature. Effective and peak phase currents, power loss, and satisfaction of the voltage constraints during commutation are some possible secondary objective functions to produce an optimal TSF. However, some of these objective functions may 
contradict others. For example, a low power loss implies fast commutation between two adjacent current phases and may cause voltage saturation. Vujicic [9] introduced a family of TSFs by using different secondary objectives, such as power loss minimization and drive constraint consideration. However, the consideration of linear magnetic characterization and a simple torque equation may reduce drive efficiency and performance. Selecting the slope of inductance only at the linear or saturation region also has a considerable impact on the effectiveness of this method, especially for the load torque near the rated value. The balanced TSF introduced in [6] considered the commutation center at the position of $\theta_{c}^{i}$, where two adjacent phases have the same torque at the same phase current. [11], [12] suggested changing the commutation center from $\theta_{c}^{i}$ to $\theta_{c}^{\lambda}$ with the increase in speed. Turn-on and turn-off angle controls were also considered in [13] to minimize torque ripples. An analytical equation was derived in [14] to calculate the turn-on angle, and the turn-off angle was obtained from the experimental equation. A logical TSF was proposed in [8], where the outgoing phase current was dampened quickly and total torque was kept constant by torque regulation produced by the incoming phase. However, the motor is considered in the linear region, and the incoming phase current regulation is based on maximizing the phase current at the start of the commutation. Increasing peak current and power loss are the other drawbacks of this method. In the present study, a constant torque trajectory in phase current plane is obtained by the use of a completely nonlinear torque equation and by considering the saturation effects in magnetic characterization. Power loss minimization is assumed as a secondary objective function. Afterward, point selection on constant torque trajectory curves is accomplished in favor of power loss reduction for each rotor position. A novel analytic invertible function is introduced to produce a torque equation and torque sharing function in consideration of the proposed power loss minimization. A modification scheme is applied to enhance the effective speed region. The simulation and experimental results are provided to demonstrate the feasibility and effectiveness of the proposed method.

\section{THEORETICAL BACKGROUND}

\section{A. Torque Production Principle}

Conventional SRMs have a double salient structure with concentrated windings on stator poles. The torque production principle in this type of motor is based on minimizing the magnetic reluctance for flux linkage paths; this principle is different from that in other types of AC motors. Energy conversion from magnetic to mechanical type has a strong relationship with rotor position and phase current in SRM. The produced torque of each individual phase caused by the flow of $i_{k}$ at the rotor position of $\theta$ can be calculated as follows:

$$
T_{e, k}=\frac{\partial \int \lambda_{k}\left(i_{k}, \theta\right) d i_{k}}{\partial \theta} \quad k=1,2, \ldots \ldots, m
$$

where $m$ is the number of motor phases. Given the highly nonlinear and saturated nature of magnetic characterization in SRM, expressing the distinct relationship among phase flux, current, and rotor position is essential. The flux linkage of each phase can be represented by the following equation based on the corresponding phase current and rotor position [16].

$$
\lambda_{k}\left(i_{k}, \theta\right)=\lambda_{s}\left(1-e^{-i_{k} \cdot f_{k}(\theta)}\right)
$$

where $\lambda_{s}$ is the saturated flux when the brotor and stator poles are aligned properly and $f_{k}(\theta)$ can be determined as follows:

$$
\begin{aligned}
& f_{k}(\theta)=a_{0}+a_{1} \cos \left(N_{r}\left(\theta-2 \pi(k-1) / N_{s}\right)\right)+\ldots . \\
& \ldots+a_{n} \cos \left(n \cdot N_{r}\left(\theta-2 \pi(k-1) / N_{s}\right)\right)
\end{aligned}
$$

In the above equation, $N_{r}$ and $N_{s}$ are the number of rotor and stator poles, respectively. Considering Eqs. (2) and (3) and integrating Eq. (1), phase torque can be obtained by the following equation:

$$
T_{e, k}\left(i_{k}, \theta\right)=\frac{\lambda_{S}}{f_{k}^{2}(\theta)} \cdot \frac{\partial f_{k}(\theta)}{\partial \theta}\left[1-\left(1+i_{k} \cdot f_{k}(\theta)\right) e^{\left.-i_{k} \cdot f_{k}(\theta)\right] .}\right.
$$

As indicated by Eq. (4), considering nonlinear and saturation effects in the magnetic characterization of an SRM can lead to a complex calculation of phase torques. In many references, Eq. (5) is utilized to approximate phase torque that originates from the linear relationship between phase flux and current shown in Eq. (6). Eq. (7) also represents the phase voltage relation, where $R$ is the winding resistance per phase and $\lambda_{k}\left(i_{k}, \theta\right)$ displays the flux linkage that corresponds to phase current $i_{k}$ and rotor position $\theta$ for the $k^{\text {th }}$ phase.

$$
\begin{aligned}
& T_{e, k}\left(i_{k}, \theta\right)=\frac{1}{2} \frac{\partial L_{k}(\theta)}{\partial \theta} \cdot i_{k}^{2} \\
& \lambda_{k}\left(i_{k}, \theta\right)=L_{k}(\theta) \cdot i_{k} \\
& U_{k}=R \cdot i_{k}+\frac{d \lambda_{k}\left(i_{k}, \theta\right)}{d t}
\end{aligned}
$$

Considering Eq. (5), a positive phase torque can be produced when the slope of the inductance profile is positive. The current flow direction has no influence on the sign of the resultant torque. Fig. 1 illustrates the operation principle of an SRM in motoring and generating modes. Phase current begins to be excited during the positive slope of the inductance profile and is damped before entering the negative torque-generating area for operation in motoring condition. Fig. 2 shows the measured inductance profile for a $4 \mathrm{KW} \mathrm{8/6} \mathrm{SRM;} \mathrm{its} \mathrm{detailed}$ nominal specifications are provided in the Appendix.

The position where the stator and rotor poles are located so that the corresponding phase flux path has maximum reluctance is called the unaligned position. In this condition, 


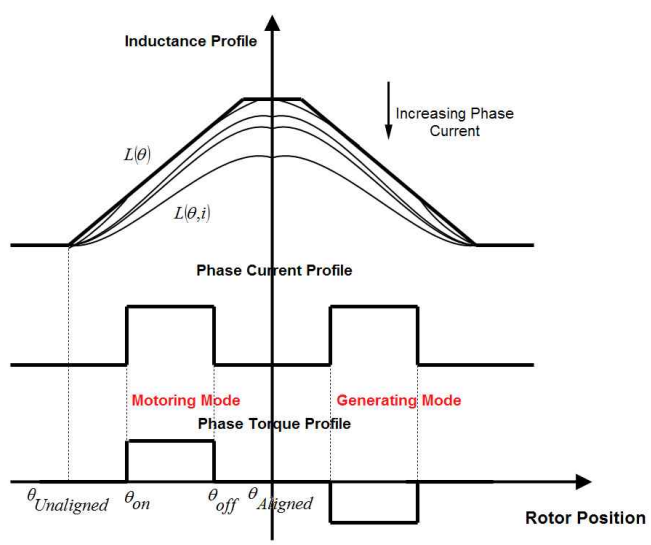

Fig. 1. Linearized and nonlinear inductance, phase current, and produced torque profile to illustrate the motoring and generating operation modes of SRM.

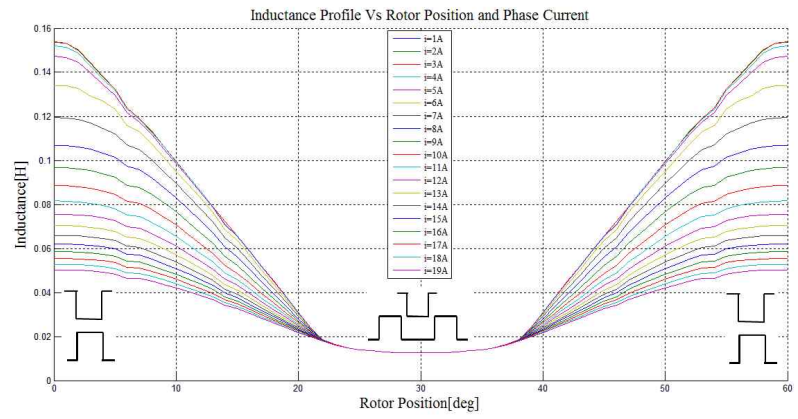

Fig. 2. Measured inductance profile for a $4 \mathrm{KW} 8 / 6$ SRM versus the rotor position for different phase currents using voltage integration method.

not only does phase inductance have the lowest value, but the slope of the inductance of its neighbor is also zero. Exciting the phase wherein the rotor position is near the unaligned position would significantly increase the phase current and would make the phase torque production negligible. Similarly, the position where the stator and rotor poles are faced together is the aligned position. Phase inductance has the maximum value in this position, and torque production capability is negligible.

\section{B. TSFS}

TSFs have been significantly considered in both research and industrial applications because of their simple concept and feasibility of implementation in torque control and ripple minimization of almost every type of SRM. In this method, torque interchange between two adjacent phases is performed in such a way that the resultant motor torque has a constant reference value. The total produced motor torque is obtained from the summation of phase torques. Generally, the conduction period of each phase is divided into two intervals: single phase conduction and two-phase conduction or commutation between adjacent phases. In the TSF method, the reference torque of the incoming phase is considered a specific function to make the resultant torque produced by the outgoing and entering phases constant. Fig. 3 presents the control block

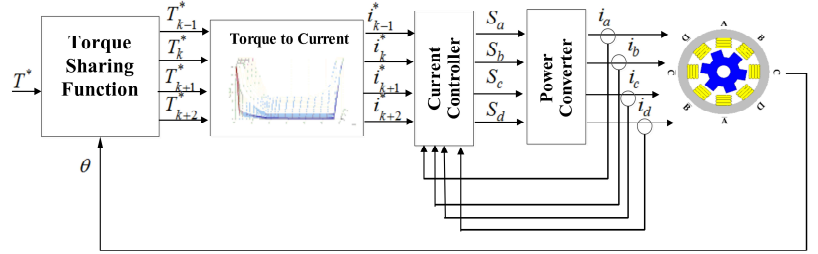

Fig. 3. Torque control block diagram utilized in TSF method for the four-phase SRM.
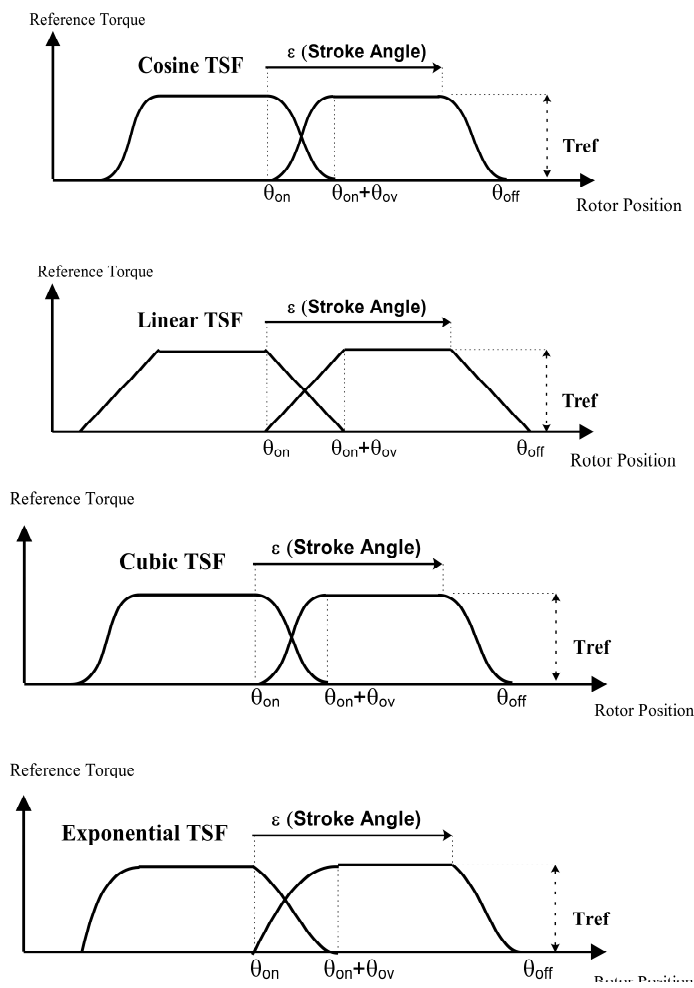

Fig. 4. Conventional TSF profiles: cosine, linear, cubic, and exponential.

diagram utilized in TSF method for the four-phase SRM.

The input reference torque is divided into individual phase torques through the TSF functions. Phase reference currents are obtained from "torque to current" look up tables at each rotor position. The PWM or hysteresis control block is applied to obtain the desired currents in conventional current control schemes. "Torque to flux" look up tables can also be employed to control the phase flux in the inner control loop. Fig. 4 shows the conventional profiles of four typical TSFs, which have linear, cosine, cubic, and exponential forms.

The reference phase torque for each phase can be generated by shifting the predefined TSFs. Phase torque commands include the zero torque region, where the corresponding phase current should be zero, and the non-zero area. This region can be divided into two major areas: commutation and single conduction mode. In the commutation area, two adjacent phases should produce positive torque based on the distinctive functions. In single conduction mode, only one phase must 
provide the reference torque. The corresponding reference phase current is obtained from the "torque to current" look up table or by the invertible SRM model [9] to determine the relevant current.

The reference torques defined for the incoming and outgoing phases during the commutation interval are computed as follows:

$$
\begin{aligned}
& T_{P h}^{*}=T_{\text {ref }} \cdot f_{\text {Linear }} \\
& f_{\text {Linear }}=\left\{\begin{array}{lc}
\frac{\left(\theta-\theta_{o n}\right)}{\theta_{o v}} & \theta_{o n} \leq \theta \leq \theta_{o n}+\theta_{o v} \\
1 & \theta_{o n}+\theta_{o v} \leq \theta \leq \theta_{o f f}-\theta_{o v} \\
\frac{\left(\theta_{o f f}-\theta\right)}{\theta_{o v}} & \theta_{o f f}-\theta_{o v} \leq \theta \leq \theta_{o f f}
\end{array}\right.
\end{aligned}
$$

where $\theta_{o n}, \theta_{o v}$, and $\theta_{o f f}$ are the start, overlap, and end of the commutation angles, respectively.

$$
\begin{aligned}
& T_{P h}^{*}=T_{\text {ref }} . f_{\text {Cosine }} \\
& f_{\text {Cosine } e}=\left\{\begin{array}{l}
\operatorname{Sin}^{2}\left(\frac{\pi}{2}\left(\frac{\theta-\theta_{o n}}{\theta_{o v}}\right)\right) \quad \theta_{o n} \leq \theta \leq \theta_{o n}+\theta_{o v} \\
\theta_{o n}+\theta_{o v} \leq \theta \leq \theta_{o f f}-\theta_{o v} \\
\operatorname{Sin}^{2}\left(\frac{\left(\theta_{o f f}-\theta\right)}{\theta_{o v}}\right) \quad \theta_{o f f}-\theta_{o v} \leq \theta \leq \theta_{o f f}
\end{array}\right. \\
& T_{P h}^{*}=T_{\text {ref }} \cdot f_{\text {Chbic }} \\
& f_{\text {Cubic }}=\left\{\begin{array}{l}
\frac{3}{\theta_{o v}^{2}}\left(\theta-\theta_{o n}\right)^{2}-\frac{2}{\theta_{o v}^{3}}\left(\theta-\theta_{o n}\right)^{3} \quad \theta_{o n} \leq \theta \leq \theta_{o n}+\theta_{o v} \\
\quad \theta_{o n}+\theta_{o v} \leq \theta \leq \theta_{o f f}-\theta_{o v} \\
1-\frac{3}{\theta_{o v}^{2}}\left(\theta-\theta_{o f f}+\theta_{o v}\right)^{2}-\frac{2}{\theta_{o v}^{3}}\left(\theta-\theta_{o f f}+\theta_{o v}\right)^{3} \quad \theta_{o f f}-\theta_{o v} \leq \theta \leq \theta_{o f f}
\end{array}\right. \\
& \begin{array}{l}
T_{P h}^{*}=T_{\text {ref }} \cdot f_{\text {Expon }} \\
f_{\text {Expon }}=\left\{\begin{array}{lc}
1-\operatorname{Exp}\left(-\frac{\left(\theta-\theta_{o n}\right)^{2}}{\theta_{o v}}\right) \quad & \theta_{o n} \leq \theta \leq \theta_{o n}+\theta_{o v} \\
\theta_{o n}+\theta_{o v} \leq \theta \leq \theta_{o f f}-\theta_{o v} & \\
\operatorname{Exp}\left(-\frac{\left(\theta_{o f f}-\theta_{o v}-\theta\right)^{2}}{\theta_{o v}}\right) & \theta_{o f f}-\theta_{o v} \leq \theta \leq \theta_{o f f}
\end{array}\right.
\end{array}
\end{aligned}
$$

Operation should be in the maximum torque per ampere (MTPA) condition to increase the efficiency of the TSF method. The torque ripple factor, which represents the quantitative index of ripples existing in the motor torque, is obtained from the following equation:

$$
T . R \%=\frac{T_{M a x}-T_{\text {Min }}}{T_{\text {ave }}} * 100 .
$$

One of the major drawbacks of TSF methods in dealing with the torque control problem and MTPA ratio is that the maximum speed where the torque ripple-free performance can be obtained is much lower than the base speed, especially at a nominal torque value. Some constraints on rising and falling times clearly cause deterioration in torque control performance. If the objective is to achieve the rated torque at the rated speed, the turn-on and turn-off angles must be changed in advance. This condition would cause a drastic decrease in torque per ampere ratio. A simple logical torque sharing function was proposed in [8]; the function decreases the outgoing current and regulates the total produced torque by controlling the incoming phase current. However, point selection for the reference currents cannot guarantee the improvement in efficiency and reduction in power loss. The step rise in the incoming reference current at the start of the commutation region can also increase the peak value of the phase current and voltage stress, especially at low-speed operation. In this study, phase current profiling is introduced by optimizing efficiency, especially at low speed, and minimizing the copper power loss caused by the higher impact of copper loss than core loss at low speed.

\section{OPTIMIZED CURRENT PROFILE AND TSF}

Copper loss in SRM is directly proportional to the square of the effective phase currents. In normal operations, each cycle of phase excitation can be divided into two parts, namely, single phase and two-phase conduction, where commutations occur. With copper loss reduction as the desired secondary objective function, Eq. (13) can be derived. Minimization of copper loss is realized by decreasing the second part in Eq. (13). Efficiency enhancement will be achieved if the first function under the integral in Eq. (13) is decreased.

$$
\begin{array}{ll}
\text { Min: } \quad & P_{C u}=R\left(I_{k}^{2}+I_{k+1}^{2}\right) \\
\text { Min : } & R \cdot\left(\frac{1}{T} \cdot \int_{\theta_{o n}}^{\theta_{o n}+\theta_{o v}}\left(i_{k}^{2}+i_{k+1}^{2}\right) d t\right)+R \cdot\left(\frac{1}{T} \cdot \int_{\theta_{o n}+\theta_{o v}}^{\theta_{o f f}-\theta_{o v}}\left(i_{k}^{2}\right) d t\right)
\end{array}
$$

Hence, the objective function can be replaced by

$$
\text { Min: } \quad i_{k}^{2}+i_{k+1}^{2} \text {. }
$$

The main target of TSFs is to control torque at a desired value, which may be changed from zero to the nominal value. If the reference torque is low, it would yield a corresponding current at a low level and SRM will operate at the linear region of its magnetic characteristics. However, SRMs are usually employed in applications with a rated value of torque to obtain high efficiency. As previously shown, almost all industrial types of SRMs operate near the nominal torque; the corresponding saturation effects cannot be ignored. The linear model for magnetic characterization and Eq. (5) for phase torque are considered to introduce the basic concept of the proposed current profile. Eq. (15) must thus be satisfied during the commutation period.

$$
T_{\text {ref }}=\frac{1}{2} \cdot \frac{\partial L_{k}(\theta)}{\partial \theta} \cdot i_{k}^{2}+\frac{1}{2} \cdot \frac{\partial L_{k+1}(\theta)}{\partial \theta} \cdot i_{k+1}^{2}
$$

The above equation denotes the elliptic curve form shown in Fig. 5 for one rotor position.

The intersection between the elliptic curves and a circle with the center of origin in the phase current plane should only be on the incoming current or outgoing current axis to obtain the minimum copper loss shown in Fig. 5. In the commutation 


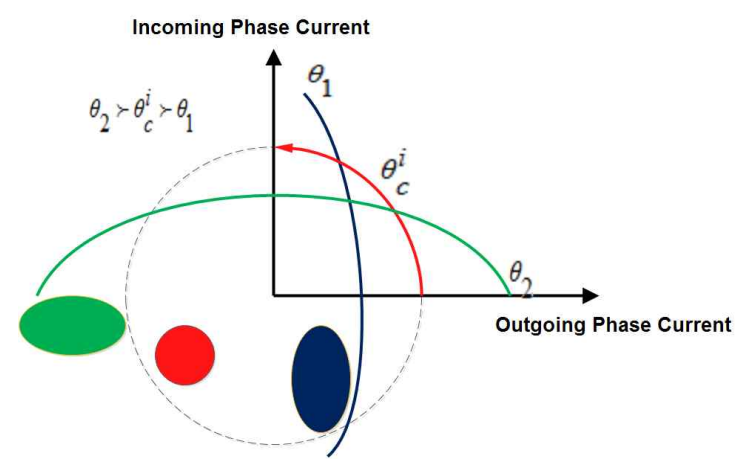

Fig. 5. Constant torque trajectory curve (elliptical curves) for one rotor position.

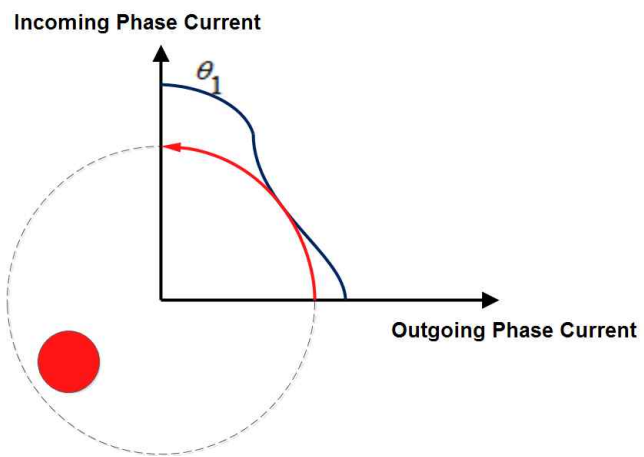

Fig. 6. Constant torque trajectory using a nonlinear SRM model and determining the optimum reference currents to achieve minimum power loss.

region, the incoming reference current increases from zero to a high value, and the outgoing reference current decreases from a high value to zero. This condition results in a phase torque tracking error in the current control loop. The motor magnetization curve shows that the slope of inductance is not only related to rotor position but also to the phase current that has a major impact on its magnitude (Fig. 2). Hence, considering Eq. (15) may cause an increase in torque ripple and decrease efficiency, especially near the rated torque value where the flux may enter in the saturation region. In this case, the problem formulation can be represented as follows:

$$
\begin{gathered}
\text { Min : } i_{k}^{2}+i_{k+1}^{2} \\
\text { subject to : } T_{r e f}=f\left(i_{k}, \theta\right)+g\left(i_{k+1}, \theta-15\right)
\end{gathered}
$$

where $f$ and $g$ are the functions that can be obtained by Eq. (4). With some approximation, the constraint equation in Eq. (16) would be similar to that in Eq. (15), whose slope of inductance is a function of the rotor position and the corresponding phase current. The elliptic curves in Fig. 5 will exhibit some variation because of the change in coefficients caused by different phase currents. Fig. 6 shows the concept of the proposed nonlinear torque sharing function and the optimized current profiling. The figure also shows that the tangent intersection of the constant torque trajectory and power loss minimization circle is on the intermediate area between the

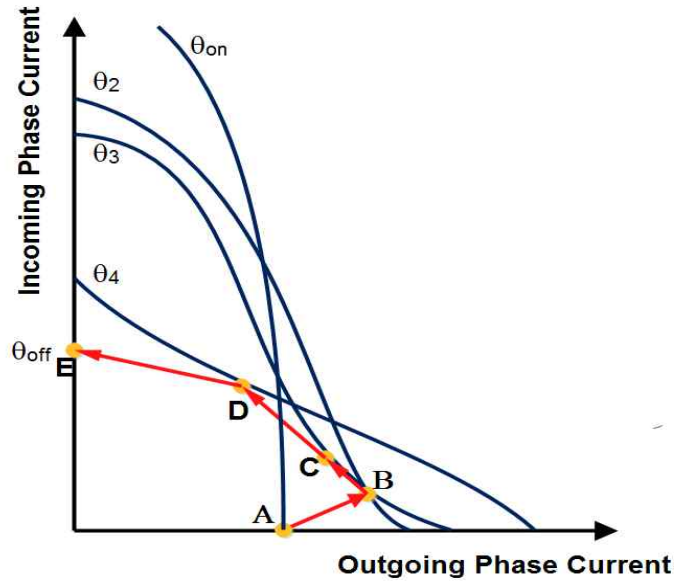

Fig. 7. Reference current selection points from the outgoing to incoming phases.

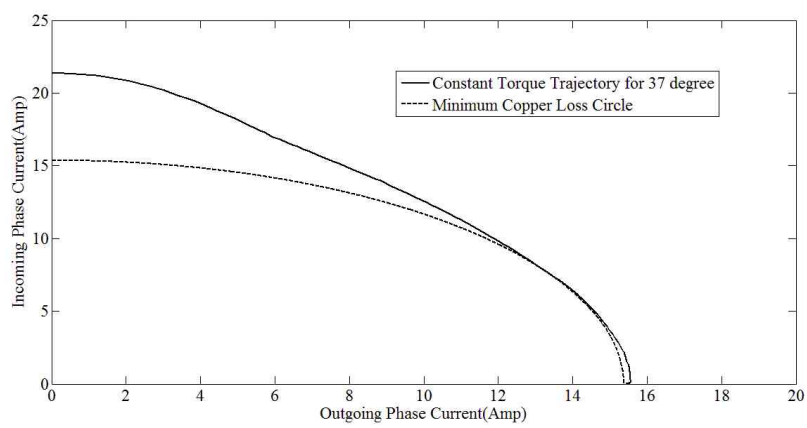

Fig. 8. Constant torque trajectory and minimum power loss circle when the rotor position is at 37 degrees and load torque is $20 \mathrm{Nm}$.

\section{$\mathrm{x}$ - and $\mathrm{y}$-axes.}

The saturation area reflects its effect on the elliptic curves so that the optimum points in the phase current plane can be out of alignment with the axes. Using this procedure for point selection on constant torque trajectories, reference currents would be smoother than those obtained by the method indicated in [8]. Fig. 7 indicates that for each rotor position, the semi-elliptic constant torque trajectories have only one intersection with the circle, a condition that indicates minimum copper loss in the area between the axes. The unique path obtained by the proposed method guarantees minimum copper loss in the commutation area because of the use of a nonlinear form of torque expression and saturation effects. The reference current profile is obtained by the proposed method for each reference torque. Fig. 8 shows the constant torque trajectory and the minimum copper loss circle when the rotor position is at 37 degrees, the unaligned position is at 30 degrees, and the alignment is at 60 degrees.

The phase current should be represented as a function of rotor position and reference torque to make this method suitable for implementation. [17] considered the phase current in each reference torque proportional to the reference current 


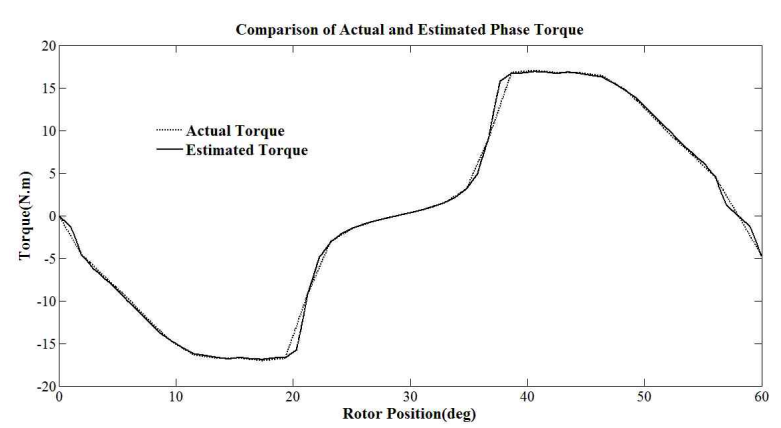

Fig. 9. Comparison of the estimated and actual phase torques for the rated phase current.

obtained from the nominal load torque. However, this method might neglect magnetic nonlinear saturation characterization. Magnetic characterization can be considered in the linear region in the light load, whereas it should be assumed as a nonlinear function near the medium and rated torque. Although the above figures show the optimization procedure, implementing this procedure requires some offline computation and high memory to store the data for each reference torque and rotor position. In this study, an analytic invertible function is introduced in Eq. (17) to express the torque equation, which represents nonlinear and saturation effects properly. Hence,

$$
T_{k}\left(i_{k}, \theta\right)=\frac{a_{k}(\theta) i_{k}^{2}}{1+b_{k}(\theta) i_{k}^{2}}
$$

where $a(\theta)$ and $b(\theta)$ are obtained from the following equations:

$$
\begin{aligned}
& a_{j}(\theta)=\sum_{n=1}^{\infty} c_{0}+c_{n} \cos \left(n N_{r} \theta-(j-1) 2 \pi / m\right) \\
& b_{j}(\theta)=\sum_{n=1}^{\infty} d_{0}+d_{n} \cos \left(n N_{r} \theta-(j-1) 2 \pi / m\right)
\end{aligned}
$$

where $j$ is the number of phases where the coefficients are calculated and $m$ is the total number of stator phases. The introduced torque function phase current can be derived from the following equation:

$$
i_{k}\left(T_{k}, \theta\right)=\sqrt{\frac{T_{k}}{a_{k}(\theta)-b_{k}(\theta) \cdot T_{k}}} .
$$

Fig. 9 presents a comparison of the estimated phase torque obtained from Eq. (17) and the actual torque from the experiments.

Copper loss minimization is realized when the effective phase currents are minimized. The effective phase current can be represented as follows:

$$
\begin{aligned}
& I_{k}^{2}=\frac{1}{\tau} \int_{\theta_{o n}}^{\theta_{o f f}} i_{k}^{2}(\theta) d \theta=\frac{1}{\tau} \int_{\theta_{o n+\theta_{o v}}}^{\theta_{o f f-\theta_{o v}}} i_{k}^{2}(\theta) d \theta+ \\
& \frac{1}{\tau} \int_{\theta_{o n}}^{\theta_{o n+\theta_{o v}}}\left[i_{k}^{2}(\theta)+i_{k}^{2}(\theta+\varepsilon)\right] d \theta
\end{aligned}
$$

The conduction of each phase can be divided into two regions: single phase conduction (where only one phase must provide the reference torque) and the commutation area (where two adjacent phases should maintain the produced torque at the desired value). Considering Eqs. (16), (20), and (21), the optimization problem can be derived as follows:

$$
\operatorname{Min}: \frac{T_{k}}{a_{k}(\theta)-b_{k}(\theta) T_{k}}+\frac{T_{k+1}}{a_{k+1}(\theta)-b_{k+1}(\theta) T_{k+1}} .
$$

Given that the total resultant torque in the commutation area should be equal to the reference level, Eq. (22) can be changed into the following representation:

$$
\begin{aligned}
& \text { Min: } \\
& \frac{T_{k}}{a_{k}(\theta)-b_{k}(\theta) T_{k}}+\frac{T_{r e f}-T_{k}}{a_{k}(\theta-\varepsilon)-b_{k}(\theta-\varepsilon)\left(T_{r e f}-T_{k}\right)}
\end{aligned}
$$

where $\varepsilon$ is the stroke angle obtained at 15 degrees for the four-phase SRM. For simplicity, the following assumptions are considered.

$$
\begin{aligned}
& a_{k}(\theta)=a \quad, b_{k}(\theta)=b \\
& a_{k+1}(\theta)=a_{k}(\theta-\varepsilon)=a^{\prime} \\
& b_{k+1}(\theta)=b_{k}(\theta-\varepsilon)=b^{\prime}
\end{aligned}
$$

The aim is to determine $T_{k}^{*}$ to minimize the objective function described by Eq. (22). This aim is achieved by fixing the derivation of Eq. (22) to zero with respect to $T_{k}$. The following equation represents the proposed TSF where commutation loss is minimized.

$$
T_{k}\left(T_{r e f}, \theta\right)=\frac{a \sqrt{a^{\prime}}-a^{\prime} \sqrt{a}+b^{\prime} \sqrt{a} \cdot T_{r e f}}{b \sqrt{a^{\prime}}+b^{\prime} \sqrt{a}}
$$

The simulation results demonstrate that the resultant TSF is suitable for use in applications with low to medium speeds and low to high torque ranges. The voltage limitation and drive converter constraints should be considered to enhance the torque ripple-free speed range of the operation. Eqs. (26) and (27) represent the voltage relationship and maximum change in the phase currents with respect to the rotor position.

$$
\begin{aligned}
& V \approx \frac{d \lambda}{d t}=\frac{\partial \lambda}{\partial \theta} \cdot \omega+\frac{\partial \lambda}{\partial i} \cdot \frac{\Delta i}{\Delta t}=\frac{\partial L}{\partial \theta} i \omega+L \frac{\Delta i}{\Delta \theta} \cdot \omega \\
& \Delta i_{k, \max }\left(\theta_{n}\right)=\left[\frac{V_{d c}}{\omega}-\frac{\partial L}{\partial \theta}\left(\theta_{n}\right) \cdot i_{k}\left(\theta_{n}\right)\right]\left(\frac{\theta_{n+1}-\theta_{n}}{L\left(\theta_{n}\right)}\right)
\end{aligned}
$$

Considering that the phase inductance for the incoming region is similar to Eq. (28) as described in [14], Eq. (29) can be obtained for the maximum current change in the incoming phase.

$$
\begin{gathered}
L(\theta)=a e^{(\theta-b) / c} \\
\Delta i_{k, \max }\left(\theta_{n}\right)=\left[\frac{V_{d c}}{\omega \cdot a e^{\left(\left(\theta_{n}-b\right) / c\right)}}-\frac{\left(\theta_{n+1}-\theta_{n}\right) i_{k}\left(\theta_{n}\right)}{c}\right]
\end{gathered}
$$




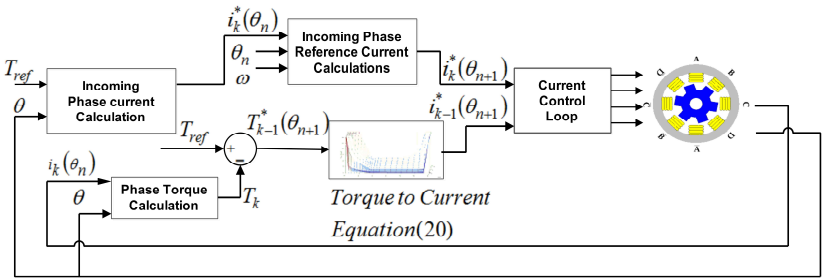

Fig. 10. Control block diagram of the proposed modification scheme.

TABLE I

Fitting Coefficient of The Proposed Torque Function

\begin{tabular}{|c|c|c|c|c|c|}
\hline$c_{0}$ & $c_{1}$ & $c_{2}$ & $c_{3}$ & $c_{4}$ & $c_{5}$ \\
\hline 0.1452 & 0.06641 & -0.087 & 0.0075 & -0.0104 & -0.028 \\
\hline$d_{0}$ & $d_{1}$ & $d_{2}$ & $d_{3}$ & $d_{4}$ & $d_{5}$ \\
\hline 0.0072 & 0.011 & 0.0072 & 0.0063 & 0.0047 & 0.0032 \\
\hline
\end{tabular}

In Eq. (28), the coefficients $a, b$, and $c$ are obtained from the curve fitting $0.0084,-1.1$, and 9.66, respectively. After calculating the reference torque for the incoming phase, the other phase is regulated based on the compensating torque error. With the proposed modification reference, the current is changed slightly in each rotor position based on the motor speed. In other words, the maximum change in phase current is limited for each rotor position because of the voltage constraint applied by the drive converter. This condition yields a torque error and reduces the efficiency of the torque control scheme. The torque error is added to the outgoing reference torque to overcome this problem similar to the proposed negative torque compensation introduced in [15]. Fig. 10 shows the block diagram of the proposed modification scheme. Instead of using a current control loop, phase torque control is used directly to avoid using the current-torque-position look up table or Eq. (20).

\section{SIMULATION AND EXPERIMENTAL RESULTS}

A $4 \mathrm{KW} 8 / 6 \mathrm{SRM}$ is employed for simulation and implementation to verify the proposed method for torque ripple minimization and commutation power loss. The detailed specifications of the SRM are provided in the Appendix. The torque sharing functions are obtained, and the optimum current profiling for each reference torque is derived with Eq. (25). The optimum phase current without modification can be obtained by considering Eqs. (18) and (19) and the coefficient provided in Table I. The regulation of the outgoing phase torque based on the torque error in the incoming phase torque tracking control loop is ignored. Fig. 11 shows the simulation results for the phase currents, fluxes, torques, and motor resultant torque at $20 \mathrm{Nm}$ load torque and at the speed of 200 rpm.
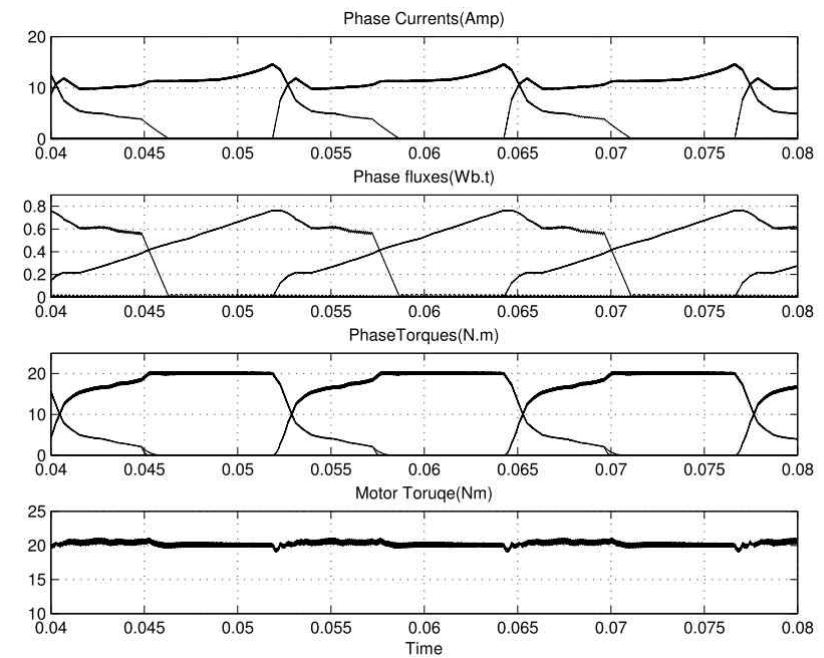

Fig. 11. Phase currents, fluxes, torques, and motor resultant torque with the proposed method and without modification at $20 \mathrm{Nm}$ load torque and at the speed of $200 \mathrm{rpm}$.

TABLE II

POWER LOSS COMPARISON WITH 20 NM LOAD TORQUE

\begin{tabular}{|c|c|c|c|c|c|}
\hline Type & Lin & Cos & Cub & Exp & Proposed \\
\hline Power Loss (W) & 28.5 & 28 & 28.2 & 28.7 & 27.1 \\
\hline
\end{tabular}

Table II presents the power loss during commutation for linear, cosine, cubic, and exponential forms and the proposed TSFs with the same condition as in Fig. 11. The results in this table provide some insights and verify power loss minimization based on the proposed method.

Voltage saturation and constraints should be considered when speed is increased because of the existing limitation in the changing rate of phase fluxes, as Eq. (26) illustrates. Fig. 12 shows the simulation results for the speed of $500 \mathrm{rpm}$, in which the converter voltage cannot provide the required flux. The changing rate of the incoming phase and torque error in this phase leads to high torque ripples in the motor resultant torque.

The modification scheme is applied by compensating for the torque error in the incoming phase with the outgoing phase such that the resultant torque remains constant. Fig. 13 presents the results for the same load torque at the speed of $1000 \mathrm{rpm}$. In the figure, the torque ripple factor (TRF) is less than $15 \%$ when the proposed modification method is employed; the measured value of the uncompensated linear and cosine TSFs are $56 \%$ and $48 \%$, respectively. Fig. 14 shows the torque speed characteristic for linear, cosine, and the proposed TSFs. The figure reveals the superiority of the proposed modified method in enhancing the torque ripple-free operational speed. Fig. 15 as well as Tables III and IV present the copper loss per phase and efficiency obtained by the proposed TSF and linear and cosine TSFs. In the figure, the proposed scheme leads to low copper loss and high efficiency, especially in high-torque and low-speed applications when iron loss is ignored. Linear and 

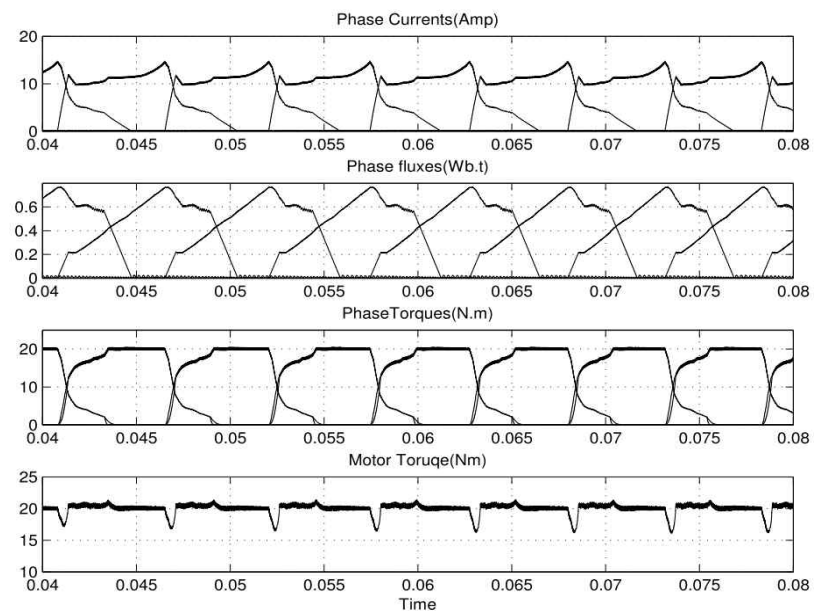

Fig. 12. Phase currents, fluxes, torques, and motor resultant torque with the proposed method and without modification at $20 \mathrm{Nm}$ load torque and at the speed of $500 \mathrm{rpm}$.
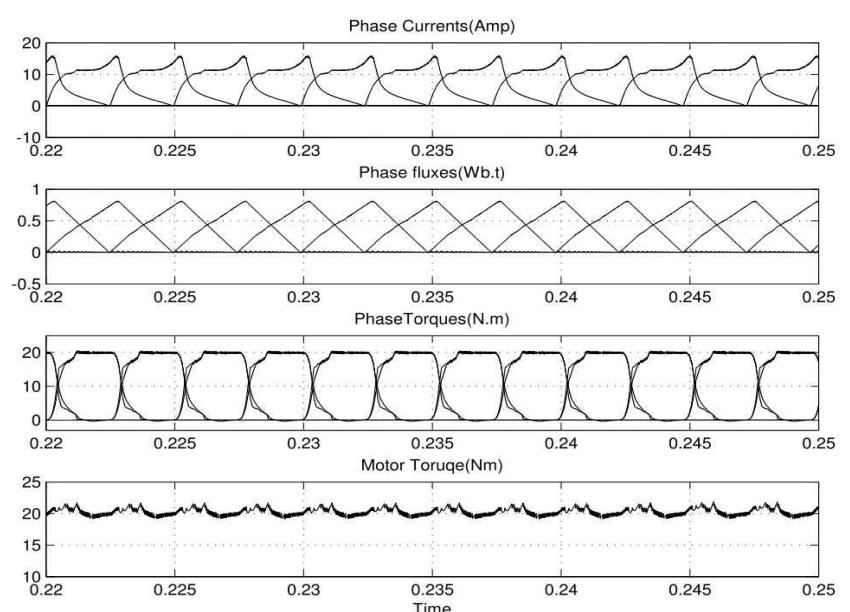

Fig. 13. Phase currents, fluxes, torques, and motor resultant torque with the proposed method and with modification at $20 \mathrm{Nm}$ load torque and at the speed of $1000 \mathrm{rpm}$.

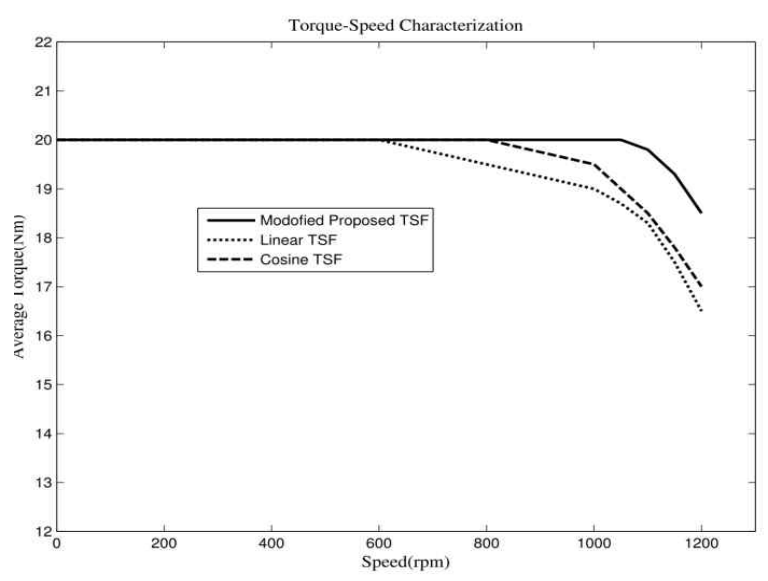

Fig. 14. Torque speed characteristics of linear, cosine, and the modified proposed TSFs.

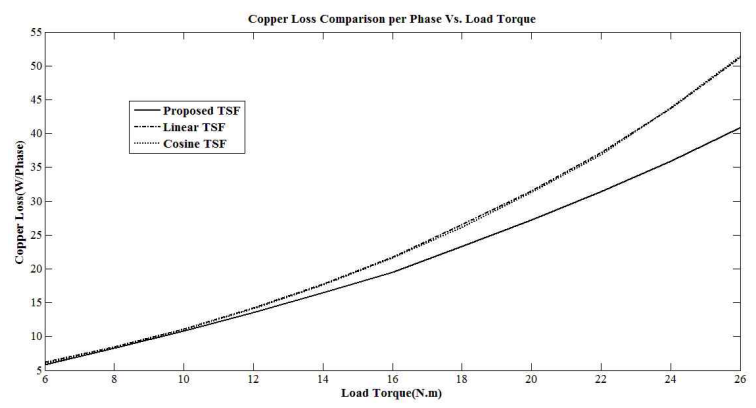

Fig. 15. Phase copper loss comparison using the proposed, linear, and cosine TSFs with respect to increasing load torque.

TABLE III

COMPARISON OF COPPER POWER LOSS OBTAINED BY LINEAR, Cosine, ANd Proposed TSFs Versus LoAd TORQue

\begin{tabular}{|c|c|c|c|c|}
\hline \multicolumn{5}{|c|}{ Power Loss (Watt/Phase) } \\
\hline Torque $(\mathrm{Nm})$ & Load \% & Linear & Cosine & Proposed \\
\hline 6 & 23 & 6.22 & 5.98 & 5.8 \\
\hline 8 & 31 & 8.47 & 8.3 & 8.28 \\
\hline 10 & 38 & 11.15 & 11 & 10.77 \\
\hline 12 & 46 & 14.2 & 14.12 & 13.5 \\
\hline 14 & 54 & 17.77 & 17.68 & 16.5 \\
\hline 16 & 61 & 21.7 & 21.65 & 19.6 \\
\hline 18 & 70 & 26.5 & 26.15 & 23.32 \\
\hline 20 & 77 & 31.5 & 31.3 & 27.24 \\
\hline 22 & 85 & 37.2 & 36.9 & 31.45 \\
\hline 24 & 92 & 43.75 & 43.82 & 35.95 \\
\hline 26 & 100 & 51.2 & 51.4 & 40.9 \\
\hline
\end{tabular}

cosine TSFs have approximately the same power loss versus the load torque. However, cosine TSF has slightly smaller losses compared with the linear type.

Table IV shows that the proposed method has high efficiency of up to $4 \%$ at low-speed and high-torque applications. The introduced modified method is implemented on an SRM rig. The main controller for implementing the proposed scheme is designed with TMS320F28335 from Texas Instruments. Phase currents are used as feedback signals and 10-bit digital signals from the absolute encoder by $1024 \mathrm{ppr}$. The analytical description of torque and phase current as introduced in Eqs. (17) and (20) as well as modification rules are programmed into DSP. Phase torques are considered feedback in the inner control loop, and the switching frequency is $10 \mathrm{kHz}$. Flux linkage data are calculated with voltage integration method. A rectangle voltage is applied to the phase at a specific rotor position. Voltage, current, and rising time are recorded with an oscilloscope. These data are used to calculate torque, flux, and the inductance profile. This procedure is 
TABLE IV

COMPARISON OF THE EFFICIENCY OF LINEAR, COSINE, AND PROPOSED TSFS VERSUS LOAD TORQUE

\begin{tabular}{|c|c|c|c|c|}
\hline \multicolumn{5}{|c|}{ Efficiency } \\
\hline Torque $(\mathrm{Nm})$ & Load \% & Linear & Cosine & Proposed \\
\hline 6 & 23 & 0.83 & 0.84 & 0.84 \\
\hline 8 & 31 & 0.83 & 0.83 & 0.83 \\
\hline 10 & 38 & 0.82 & 0.82 & 0.82 \\
\hline 12 & 46 & 0.81 & 0.81 & 0.82 \\
\hline 14 & 54 & 0.80 & 0.80 & 0.81 \\
\hline 16 & 61 & 0.79 & 0.79 & 0.81 \\
\hline 18 & 70 & 0.78 & 0.78 & 0.80 \\
\hline 20 & 77 & 0.76 & 0.76 & 0.79 \\
\hline 22 & 85 & 0.75 & 0.75 & 0.78 \\
\hline 24 & 92 & 0.74 & 0.74 & 0.77 \\
\hline 26 & 100 & 0.72 & 0.72 & 0.76 \\
\hline
\end{tabular}

repeated from the unaligned to the aligned position by the step of 1 degree in the rotor position. Fig. 16 shows the experimental results for the $10 \mathrm{Nm}$ load torque without modification using the proposed scheme.

In the figure, phase voltage is considered low enough to reveal the converter saturation effect on the rising phase torque in the incoming phase and decrease the resultant torque. The phase tracking error in the outgoing phase also tipped the shape of the motor resultant torque. In this case, the phase voltage is fixed to 150 V. Fig. 17 presents the same situation with the modification scheme. The torque ripple in the motor torque is reduced, and the average torque remains at $10 \mathrm{Nm}$

Fig. 18 shows the experimental results for the $14 \mathrm{Nm}$ load torque without modification using the proposed scheme. Fig. 19 presents the same conditions with the proposed modification method. In this figure, the torque error in the incoming phase is added to the reference phase torque of the outgoing phase. The excess torque in the outgoing phase decreases the reference torque in the incoming phase. Fig. 20 shows the reference torque calculated from Eq. (25) for the load torque of $14 \mathrm{Nm}$. Fig. 21 shows the modified TSF utilized to maintain the total torque in the commutation region. Fig. 19 shows the decrease in TRF in the motor resultant torque compared with Fig. 18. The measured TRF for the modified TSF in Fig. 19 is $41 \%$, and the ripple factor index is $72 \%$ without modification in Fig. 18.

\section{CONCLUSIONS}

A torque-sharing function was proposed in this study in consideration of nonlinear and magnetic saturation effects. Semi-elliptic curves that correspond to constant torque were introduced for different rotor positions. The constant torque

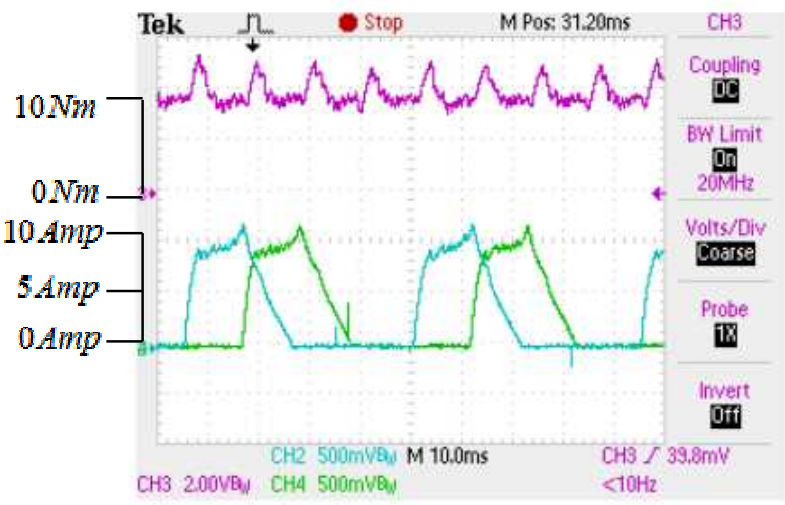

Fig. 16. Motor torque and phase currents for the proposed scheme without modification at load torque of $10 \mathrm{Nm}$ and speed of 250 rpm.

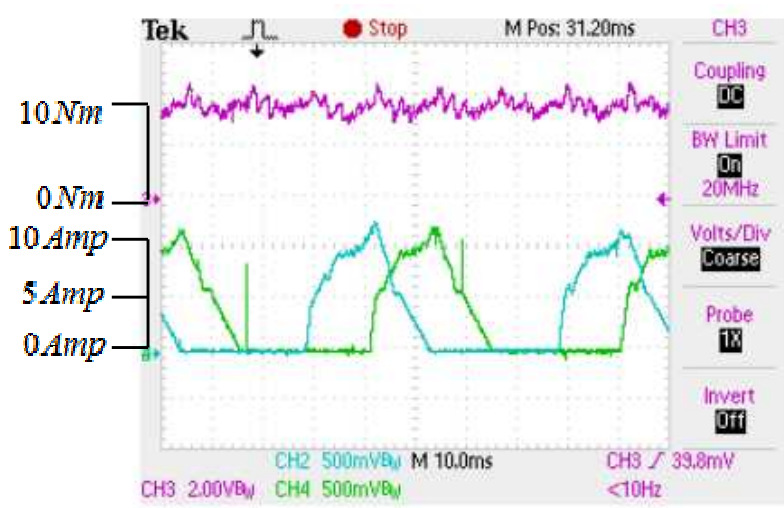

Fig. 17. Motor torque and phase currents for the proposed scheme with modification at load torque of $10 \mathrm{Nm}$ and speed of $250 \mathrm{rpm}$.

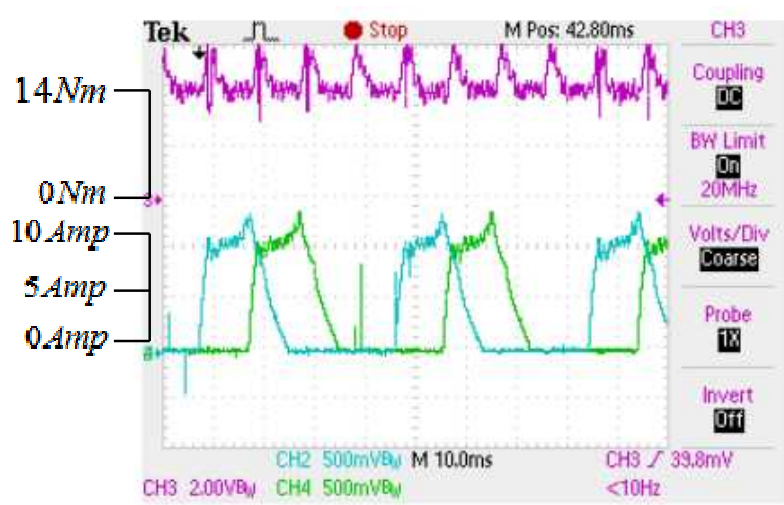

Fig. 18. Motor torque and phase currents for the proposed scheme without modification at load torque of $14 \mathrm{Nm}$, speed of $250 \mathrm{rpm}$, and $150 \mathrm{~V}$ phase voltage.

trajectories only have one tangent intersection with the copper loss minimization circle on the incoming and outgoing phase planes. The reference current paths were obtained for an industrial type, four-phase $8 / 6 \mathrm{SRM}$ with the nonlinear function of reference torque and rotor position. A novel analytic invertible function was introduced to represent phase torque and current relationships based on the rotor position. An online modification method was presented to improve the 


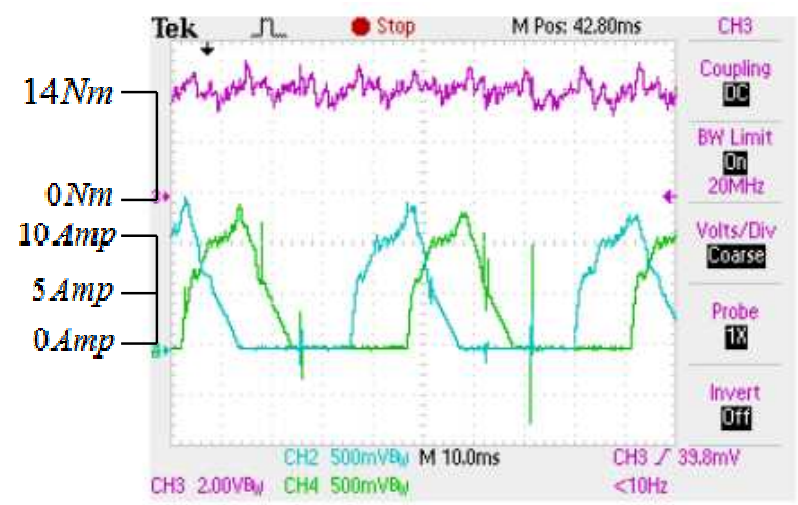

Fig. 19. Motor torque and phase currents for the proposed scheme with modification at load torque of $14 \mathrm{Nm}$, speed of $250 \mathrm{rpm}$, and $150 \mathrm{~V}$ phase voltage.

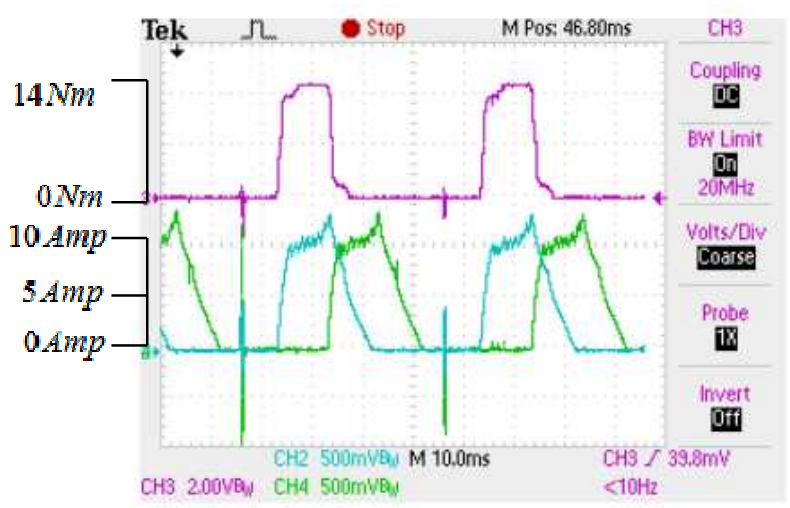

Fig. 20. Reference phase torque and phase currents for the proposed scheme without modification at load torque of $14 \mathrm{Nm}$, speed of $250 \mathrm{rpm}$, and $150 \mathrm{~V}$ phase voltage.

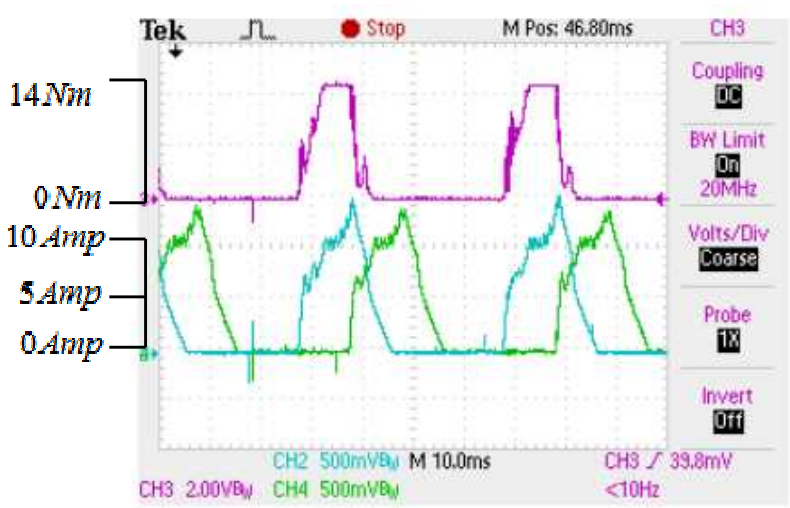

Fig. 21. Reference phase torque and phase currents for the proposed scheme with modification at load torque of $14 \mathrm{Nm}$, speed of $250 \mathrm{rpm}$, and $150 \mathrm{~V}$ phase voltage.

performance of the proposed scheme when the speed is increased. The method satisfies the voltage limitation constraint based on the motor speed and retains the constant torque. The simulation and experimental results demonstrate the effectiveness of the proposed TSF and confirm the superiority of the novel method compared with conventional
TSF types in terms of power loss minimization and torque ripple reduction. The comparisons were performed by optimal TSFs for linear and cosine types from computer simulations at different turn-on and turn-off angles. The proposed TSF exhibits good performance in torque speed characteristics, improves drive efficiency, and is capable of adapting to different motor speeds.

\section{APPENDIX}

SR MOTOR PARAMETERS

\begin{tabular}{|c|c|c|c|}
\hline Stator Poles & 8 & Rotor Poles & 6 \\
\hline Rated Power & $4 \mathrm{KW}$ & Rated Speed & $1500 \mathrm{rpm}$ \\
\hline Rated Voltage & $\begin{array}{c}280 \\
\text { V/phase }\end{array}$ & $\begin{array}{c}\text { Rated Phase } \\
\text { Current }\end{array}$ & $18 \mathrm{Amp}$ \\
\hline $\begin{array}{c}\text { Phase } \\
\text { Resistance }\end{array}$ & $0.7 \Omega$ & Rated Torque & $26 \mathrm{Nm}$ \\
\hline
\end{tabular}

\section{REFERENCES}

[1] S. K. Sahoo, S. Dasgupta, S. K. Panda, and J. X. Xu, “A lyapunov function based robust direct torque controller for switched reluctance motor drive system," IEEE Trans. Power Electron., Vol. 27, No. 2, pp. 555-564, Feb. 2012.

[2] R. Krishnan, Switched Reluctance Motor Drives Modeling, Simulation, Analysis, Design and Applications, Boca Rotan, CRC Press, 2001.

[3] Y. K. Choi, H. S. Yoon, and C. S. Koh, "Pole-shaped optimization of a switched reluctance motor for torque ripple reduction," IEEE Trans. Magn., Vol. 43, No. 4, pp. 1797-1800, Apr. 2007.

[4] S. I. Nebeta, I. E. Chabu, L. Lebensztajn, D. A. P. Correa, W. M. Dasilva, and K. Hameyer, "Mitigation of the torque ripple of a switched reluctance motor through a multi objective optimization," IEEE Trans. Magn., Vol. 44, No. 6, pp. 1018-1021, Jun. 2008.

[5] M. Illic-spong, T. J. E. Miller, S. R. MacMinn, and J. S. Thorp, "Instantaneous torque control of electric motor drives," IEEE Trans. Power Electron., Vol. 2, No. 1, pp. 55-61, Jan. 1987.

[6] R. S. Wallace and D.G. Taylor, "A balanced commutatorfor switched reluctance motors to reduce torque ripple," IEEE Trans. Power Electron., Vol. 7, No. 4, pp. 617-626, Oct. 1992.

[7] I. Husain, "Minimization of torque ripples in SRM drives," IEEE Trans. Ind. Electron., Vol. 49, No.1, pp. 28-39, Feb. 2002.

[8] D. H. Lee, J. Liang, Z. G. Lee, and J. W. Ahn, “A simple nonlinear logical torque sharing function for low toruqe ripple SR drives," IEEE Trans. Ind. Electron., Vol. 56, No. 8, pp. 3021-3028, Aug. 2009.

[9] V. P. Vujicic, "Minimization of torque ripple and copper losses in switched reluctance drive," IEEE Trans. Power Electronics, Vol. 27, No. 1, pp. 388-399, Jan. 2012.

[10] N. H. Fuengwarodsakul, M. Menne, R. B. Inderka, and R. W. DeDoncker, "High dynamic four quadrant switched reluctance drive based on DITC," IEEE Trans. Ind. Appl., Vol. 41, No. 5, PP. 1232-1242, Sep. 2005.

[11] P. C. Kjaer, J. J. Gribble, and T. J. E. Miller, "High-grade control of switched reluctance machines," IEEE Trans. Ind. 
Appl., Vol. 33, No. 6, pp. 1585-1593, Nov./Dec. 1997.

[12] I. Husain, "Minimization of torque ripple in SRM drives," IEEE Trans. Ind. Electron., Vol. 49, No. 1, pp. 28-39, Feb. 2002.

[13] Y. Sozer and D. A. Torrey, "Optimal turn-off angle control in the face of automatic turn-on angle control for switched-reluctance motors," IET Electr. Power Appl., Vol. 1, No. 3, pp. 395-401, May 2007.

[14] Y. Xu, Z. Zhong, R. Chen, and L. S. L. Lu, "Analytical method to optimise turn-on angle and turn-off angle for switched reluctance motor drives," IET Electr. Power Appl., Vol. 6, No. 9, pp. 593-603, Nov. 2012.

[15] D. H. Lee, S. Y. Ahn, and J. W. Ahn, "A simple negative torque compensation scheme for a high speed switched reluctance motor," Journal of Power Electronics, Vol. 12, No. 1, pp. 58-66, Jan. 2012.

[16] M. Spong, R. Marino, S. Peresada, and D. Taylor, "Feedback linearizing control of switched reluctance motor," IEEE Trans. Automat. Contr., Vol. AC-32, No. 5, PP. 371-379, May 1987.

[17] F. Filicori, C. G. L., Bianco, and A. Tonielli, "Modeling and control strategies for a variable reluctance direct-drive motor," IEEE Trans. Ind. Electronics, Vol. 40, No. 1, pp. 105-115, Feb. 1993.

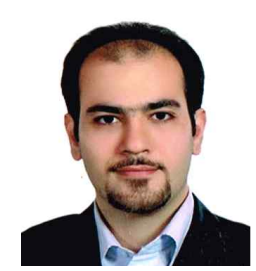

Milad Dowlatshahi was born in Isfahan, Iran, in 1984. He received his B.S. and M.S degrees in electrical engineering from Isfahan University of Technology, Isfahan, Iran, in 2006 and 2009, respectively. He is currently pursuing his Ph.D. degree in electrical engineering. His research interests include electric motor drives, power electronics, and SRM design and control. He was a visiting researcher at the Smart Mechatronics Lab of Kyungsung University, Busan, Korea, from July 2012 to January 2013.

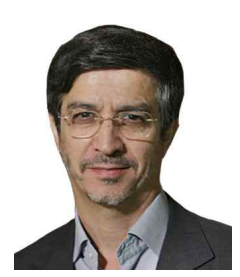

Sayed Morteza Saghaiannejad was born in Isfahan, Iran, in 1952. He received his B.S., M.S., and Ph.D. degrees in electrical engineering from the University of Kentucky, USA, in 1977, 1979, and 1989, respectively. $\mathrm{He}$ has been a faculty member of Isfahan University of Technology since 1979 and is currently a professor at the Electrical and Computer Engineering Department of the said institution. He is the author and co-author of various journal and conference papers. His current research interests are in the areas of electrical machines, power electronics, and advanced motor drive systems.

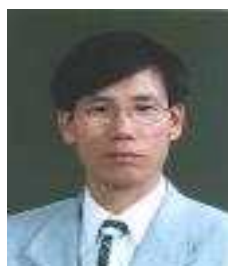

Jin-Woo Ahn was born in Busan, Korea, in 1958. He received his B.S., M.S., and Ph.D. degrees in electrical engineering from Pusan National University, Pusan, Korea, in 1984, 1986, and 1992, respectively. He has been with Kyungsung University, Busan, Korea, as a professor in its Department of Mechatronics Engineering since 1992. He is the author of five books on SRM and more than 120 research papers. He also has more than 20 patents. His current research interests are in the areas of advanced motor drive systems and electric vehicle drives. $\mathrm{He}$ is currently serving as the editor-in-chief of the Journal of International Conference on Electric Machines and Systems and the general chairman of ICEMS 2013 and IEEE/ICIT 2014.

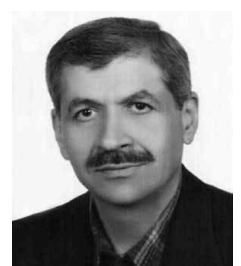

Mehdi Moallem received his Ph.D. degree in electrical engineering from Purdue University, West Lafayette, Indiana, USA in 1989. He is currently a full professor at the Department of Electrical and Computer Engineering, Isfahan University of Technology, Isfahan, Iran. He is the author and co-author of various journal and conference papers. His research interests include design and optimization of electromagnetic devices, application of advance numerical techniques and expert systems to the analysis and design of electrical machines, and power quality. $\mathrm{He}$ is a recipient of many national and international awards. 